\title{
QUASI-FREE RESOLUTIONS OF HILBERT MODULES
}

\author{
RONALD G. DOUGLAS AND GADADHAR MISRA
}

\begin{abstract}
The notion of a quasi-free Hilbert module over a function algebra $\mathcal{A}$ consisting of holomorphic functions on a bounded domain $\Omega$ in complex $m$ space is introduced. It is shown that quasi-free Hilbert modules correspond to the completion of the direct sum of a certain number of copies of the algebra $\mathcal{A}$. A Hilbert module is said to be weakly regular (respectively, regular) if there exists a module map from a quasi-free module with dense range (respectively, onto). A Hilbert module $\mathcal{M}$ is said to be compactly supported if there exists a constant $\beta$ satisfying $\|\varphi f\| \leq \beta\|\varphi\|_{X}\|f\|$ for some compact subset $X$ of $\Omega$ and $\varphi$ in $\mathcal{A}, f$ in $\mathcal{M}$. It is shown that if a Hilbert module is compactly supported then it is weakly regular. The paper identifies several other classes of Hilbert modules which are weakly regular. In addition, this result is extended to yield topologically exact resolutions of such modules by quasi-free ones.
\end{abstract}

\section{INTRODUCTION}

While the study of linear operators on Hilbert space goes back more than a hundred years, attempts at understanding more than one operator are of more recent origin. Rings of operators were investigated in the celebrated series of papers by Murray and von Neumann [30, 31, 32, 34 in the thirties, but that study led to the development of operator algebras. This subject is somewhat different than operator theory, and most recently has led to noncommutative geometry [10]. Also, there have been several approaches to non-selfadjoint multivariate operator theory. For example, there is the study of non-selfadjoint operator algebras which was initiated by Kadison and Singer [29] and has been developed by many authors over the years (cf. [14]). In [4], 5], Arveson extended results on function algebras, especially the disk algebra, to non-selfadjoint subalgebras of $C^{*}$-algebras. There is the study of operators in various concrete settings, usually defined on spaces of holomorphic functions such as the Hardy and Bergman spaces (cf. 17, 36]). Generalizations of operators on Hardy space have been undertaken by several authors recently including Arveson [6], Popescu [38, and Davidson [15]. Finally, there is the module approach (cf. [24]), in which an algebraic point of view is emphasized and the extension of techniques from algebraic and complex geometry is the key. This note makes a contribution to the latter program.

In commutative algebra a principal object of study is the collection of modules over a given ring. While in most instances the collection has additional structure and forms a semigroup, a group or even a ring, one way to study individual modules is by relating them to simpler ones. If the ring is Noetherian, then one uses projective modules which can be characterized in this context as submodules of free modules. The latter are the direct sum of copies of the ring. Otherwise, one uses a different class of modules as building blocks. The techniques for studying general modules in terms of the simpler ones is via a resolution.

The research of both the authors was supported in part by DST - NSF S\&T Cooperation Programme. This paper was completed while the first named author was visiting the Fields Institute on a development leave from Texas A \& M University. 
In this paper we propose the class of "quasi-free Hilbert modules" as the building blocks for the general ones. We discuss the Sz.-Nagy-Foias model [33] in terms of resolutions recalling that this interpretation was a key motivation for the module approach to multivariate operator theory. Then we show under reasonably general hypotheses, involving the support of the module in some sense, the existence of a topologically exact resolution by quasi-free Hilbert modules. We discuss various characterizations of the class of modules possessing such resolutions. Finally, we describe some uses of resolutions, that is, how one can extract information and invariants for a Hilbert module from resolutions.

1.1. Hilbert Modules. Let $\Omega$ be a bounded domain in $\mathbb{C}^{m}$. Examples are the unit ball, $\mathbb{B}^{m}$, the polydisk, $\mathbb{D}^{m}$, or any strongly pseudo-convex domain $\Omega$ in $\mathbb{C}^{m}$. Of course, there are also many examples for which the boundary, $\partial \Omega$, is not nice. Nonetheless, we will consider the natural function algebra $\mathcal{A}(\Omega)$ obtained from the closure in the supremum norm on $\Omega$ of all functions holomoprhic in some neighborhood of the closure of $\Omega$. If $\partial \Omega$ is not nice, there may be other natural algebras, perhaps generated by the polynomials or rational functions with poles outside the closure of $\Omega$. For more refined results, one will probably need to make additional assumptions about the boundary but we will not need to do that in this paper. For $\Omega=\mathbb{B}^{m}$ or $\mathbb{D}^{m}$, one obtains the familiar ball algebra or the poly-disk algebra.

A Hilbert module $\mathcal{M}$ over $\mathcal{A}(\Omega)$ is a Hilbert space with a multiplication $\mathcal{A}(\Omega) \times \mathcal{M} \rightarrow \mathcal{M}$ making $\mathcal{M}$ into a unital module over $\mathcal{A}(\Omega)$ and such that multiplication is continuous. Using the closed graph theorem one can show the existence of a constant $\alpha$ such that

$$
\|\varphi f\|_{\mathcal{M}} \leq \alpha\|\varphi\|_{\mathcal{A}(\Omega)}\|f\|_{\mathcal{M}} .
$$

One says that $\mathcal{M}$ is a contractive Hilbert module if $\alpha=1$. Classical examples of contractive Hilbert modules are

(i) the Hardy module $H^{2}\left(\mathbb{D}^{m}\right)$ (over the algebra $\mathcal{A}\left(\mathbb{D}^{m}\right)$ ) which is the closure of the polynomials, $\mathbb{C}[\underline{z}]$, in $L^{2}\left(\partial D^{m}\right)$ and

(ii) the Bergman module, $B^{2}(\Omega)$ (over the algebra $\mathcal{A}(\Omega)$ ) which is the closure of $\mathcal{A}(\Omega)$ in $L^{2}(\Omega)$ with volume measure on $\Omega$.

The simplest family of modules over $\mathcal{A}(\Omega)$ corresponds to evaluation at a point in the closure of $\Omega$. For $\underline{z}$ in the closure of $\Omega$, we make the one-dimensional Hilbert space $\mathbb{C}$ into the Hilbert module $\mathbb{C}_{\underline{z}}$, by setting $\varphi v=\varphi(z) v$ for $\varphi \in \mathcal{A}(\Omega)$ and $v \in \mathbb{C}$. If $\mathcal{M}$ and $\mathcal{N}$ are Hilbert modules over $\mathcal{A}(\Omega)$, then there are two obvious ways to make the Hilbert space tensor product $\mathcal{M} \otimes \mathcal{N}$ into a module over $\mathcal{A}(\Omega)$. One obtains the module tensor product, $\mathcal{M} \otimes_{\mathcal{A}(\Omega)} \mathcal{N}$, by requiring that the multiplication on $\mathcal{M}$ and the multiplication on $\mathcal{N}$ are equal, that is, one takes the closed submodule $\mathcal{S}$ of $\mathcal{M} \otimes \mathcal{N}$ generated by the elements $\varphi f \otimes g-f \otimes \varphi g$, for $f \in \mathcal{M}, g \in \mathcal{N}$, and $\varphi \in \mathcal{A}(\Omega)$ and defines $\mathcal{M} \otimes_{\mathcal{A}(\Omega)} \mathcal{N}$ to be the quotient $\mathcal{M} \otimes \mathcal{N} / \mathcal{S}$ on which the two multiplications agree.

We use the module tensor product to accomplish localization of a Hilbert module $\mathcal{M}$ over $\mathcal{A}(\Omega)$ by considering $\mathcal{M} \otimes_{\mathcal{A}(\Omega)} \mathbb{C}_{\underline{z}}$ which is isomorphic to the direct sum of $k$ copies of $\mathbb{C}_{\underline{z}}$, where $k$ can be any cardinal number from zero to the module rank of $\mathcal{M}$. Not only do we consider these tensor products at each point of the closure of $\Omega$, but together they form the spectral sheaf, $\operatorname{Sp}(\mathcal{M})$, of the module $\mathcal{M}$. We will let $\operatorname{Sp}_{\underline{z}}(\mathcal{M})$ denote $\mathcal{M} \otimes_{\mathcal{A}(\Omega)} \mathbb{C}_{\underline{z}}$. The spectral sheaf $\operatorname{Sp}(\mathcal{M})$ can be the zero sheaf but, in general, it consists of the direct sum of a number of copies of $\mathbb{C}_{\underline{z}}$ over each point $\underline{z}$ in the closure of $\Omega$. If the spectral sheaf $\operatorname{Sp}(\mathcal{M})$ defines a holomorphic hermitian bundle over $\Omega$, then we say that $\mathcal{M}$ is locally free. The 
spectral sheaf of the Hardy or Bergman modules is a hermitian holomorphic line bundle over $\Omega$.

There have been two main lines of research concerning Hilbert modules, one studying submodules and the other quotient modules. Using the classical theorem of Beurling 8 on invariant subspaces of the unilateral shift operator, one can show that all nontrivial submodules of the Hardy module $H^{2}(\mathbb{D})$ over the disk algebra $\mathcal{A}(\mathbb{D})$ are isometrically isomorphic. The Rigidity Theorem ([24], 25]) shows that the situation in higher dimensions is very different. Two submodules defined by taking the closure of ideals in $\mathbb{C}[\underline{z}]$ in the Hardy or Bergman modules (and other more general modules), which satisfy certain hypotheses, are similar or even quasi-similar if and only if the ideals are the same. Thus the rigidity, the closures cannot be equivalent in any reasonable sense unless the ideals are equal. The hypotheses eliminate principal ideals and insure that the zero sets of the associated primary ideals intersect $\Omega$. The result demonstrates that there is a great variety of non equivalent Hilbert modules in the higher dimensional setting. The proof relies on a higher order generalization of the spectral sheaf and rests on results from commutative algebra.

The work on quotient modules concerns relating properties of a quotient module with those of the submodule in cases where the latter consists of functions that vanish to some order in the normal direction to a hypersurface. Again subject to mild hypotheses, one characterizes (cf. [22, [21]) the quotient module in terms of the local geometry of the hypersurface and the spectral sheaf of the larger module.

1.2. Sz.-NAGY-Foias Model. One powerful approach to the study of contraction operators on a complex Hilbert space is the model theory of Sz.-Nagy and Foias [33]. To understand the interpretation of their model in the module context, we must first recall the theorem of von Neumann [35] which states for a contraction operator $T$ on a Hilbert space $\mathcal{H}$ and a polynomial $p$ we have $\|p(T)\| \leq\|p\|_{\mathcal{A}(D)}$. This inequality enables one to make $\mathcal{H}$ into a contractive Hilbert module over $\mathcal{A}(D)$. Thus, contraction operators on Hilbert space and contractive Hilbert modules are two ways of looking at the same thing.

The co-isometric form of the Sz.-Nagy-Foias model for contraction operators yields an isometry $W$ on a Hilbert space $\mathcal{K}=\mathcal{H} \oplus \mathcal{G}$ with $W(\mathcal{G}) \subseteq \mathcal{G}$ and such that $T=P_{\mathcal{H}} W_{\mid \mathcal{H}}$. This can also be written $0 \leftarrow \mathcal{H} \leftarrow \mathcal{K} \leftarrow \mathcal{G} \leftarrow 0$, where the arrows are module maps with the map from $\mathcal{K}$ to $\mathcal{H}$ being the orthogonal projection and the map from $\mathcal{G}$ to $\mathcal{K}$ being inclusion. This is an example of a resolution of Hilbert modules. Moreover, one has that $\mathcal{G}$ is unitarily equivalent to some vector-valued Hardy module $H_{\mathcal{E}^{*}}^{2}(\mathbb{D})$ and $\mathcal{K}$ is unitarily equivalent to $H_{\mathcal{E}}^{2}(\mathbb{D}) \oplus \mathcal{U}$, where $W$ is the shift operator on $H_{\mathcal{E}}^{2}(\mathbb{D})$ and is a unitary operator on $\mathcal{U}$. Further, one shows that $\mathcal{U}=\{0\}$ if and only if $T^{* k} \rightarrow 0$ in the strong operator topology. Such contractions are said to belong to class $C_{.0}$ by Sz.-Nagy and Foias and in this case the resolution has the simpler form $0 \leftarrow \mathcal{H} \leftarrow H_{\mathcal{E}}^{2}(\mathbb{D}) \leftarrow H_{\mathcal{E}^{*}}^{2}(\mathbb{D}) \leftarrow 0$. The modules appearing in such a resolution are the direct sum of copies of the Hardy module and all the module maps are partial isometries which makes the resolution especially nice.

No analogous results are known for the bounded case unless the module is completely bounded and hence similar to a contractive one 37]. Here we are interested in the question of when resolutions exist, not just over $\mathcal{A}(\mathbb{D})$ but for the multivariate case which we take up in the next section. 


\section{Quasi-Free Modules}

As we indicated in the introduction, a most important issue in considering resolutions of Hilbert modules is just what collection of modules to use as the building blocks. A second issue concerns the nature of the module maps. In the case of class $C_{.0}$ contractive Hilbert modules over the disk algebra, the modules used are direct sums of copies of the Hardy module and the maps are partial isometries. Moreover, the existence of such a resolution is based on the existence of a unitary or co-isometric dilation. Most of the early consideration of resolutions (17], 19]) followed this lead and, for example, the notion of Silov module was introduced for this reason. Now, however, constructing resolutions via such dilations seems to be the wrong approach ${ }^{1}$, at least for Hilbert modules over $\mathcal{A}(\Omega)$, when $\Omega$ lies in $\mathbb{C}^{m}$ with $m>1$. To illustrate, we consider a simple example.

If we consider $\mathbb{C}_{\underline{0}}$ over $\mathcal{A}\left(\mathbb{D}^{2}\right)$, then we have that $\mathbb{C}_{\underline{0}}$ is unitarily equivalent to $H^{2}\left(\mathbb{D}^{2}\right) / H_{\underline{0}}^{2}\left(\mathbb{D}^{2}\right)$, where $H_{\underline{0}}^{2}\left(\mathbb{D}^{2}\right)=\left\{f \in H^{2}\left(\mathbb{D}^{2}\right): f(\underline{0})=0\right\}$. Hence, $H^{2}\left(\mathbb{D}^{2}\right)$ provides a co-isometric dilation of $\mathbb{C}_{\underline{0}}$ and $L^{2}\left(\partial \mathbb{D}^{2}\right)$ is a unitary dilation of $\mathbb{C}_{\underline{0}}$ a la Ando $\underline{3}$. However, $H^{2}\left(\mathbb{D}^{2}\right)$ and $H_{\underline{0}}^{2}\left(\mathbb{D}^{2}\right)$ are not unitarily equivalent. More important, if we consider their spectral sheaves, then

$$
\operatorname{Sp}_{\underline{z}}\left(H^{2}\left(\mathbb{D}^{2}\right)\right)=H^{2}\left(\mathbb{D}^{2}\right) \otimes_{\mathcal{A}} \mathbb{C}_{\underline{z}} \cong \mathbb{C}_{\underline{z}}, \underline{z} \in \mathbb{D}^{2}
$$

and

$$
\operatorname{Sp}_{\underline{z}}\left(H_{\underline{0}}^{2}\left(\mathbb{D}^{2}\right)\right)=H_{\underline{0}}^{2}\left(\mathbb{D}^{2}\right) \otimes_{\mathcal{A}} \mathbb{C}_{\underline{z}} \cong \begin{cases}\mathbb{C}_{\underline{z}} & \underline{z} \neq \underline{0} \\ \mathbb{C}_{\underline{0}} \oplus \mathbb{C}_{\underline{0}} \underline{z}=\underline{0}\end{cases}
$$

This shows, in particular, that $H^{2}\left(\mathbb{D}^{2}\right)$ and $H_{\underline{0}}^{2}\left(\mathbb{D}^{2}\right)$ are not similar but also that $\operatorname{Sp}\left(H_{\underline{0}}^{2}\left(\mathbb{D}^{2}\right)\right)$ is not a vector bundle. Therefore, $H_{\underline{0}}^{2}\left(\mathbb{D}^{2}\right)$ is not locally free. However, the resolution:

$$
0 \longleftarrow \mathbb{C}_{\underline{0}} \longleftarrow H^{2}\left(\mathbb{D}^{2}\right) \stackrel{X}{\longleftarrow} H^{2}\left(\mathbb{D}^{2}\right) \oplus H^{2}\left(\mathbb{D}^{2}\right) \stackrel{Y}{\longleftarrow} H^{2}\left(\mathbb{D}^{2}\right) \longleftarrow 0
$$

where $(X(f \oplus g))(\underline{z})=z_{2} f(\underline{z})-z_{1} g(\underline{z})$ and $Y(f)(\underline{z})=\left(z_{1} f \oplus z_{2} f\right)(\underline{z})$, demonstrates that $\mathbb{C}_{\underline{0}}$ has a locally free resolution.

Now, we introduce the notion of a quasi-free Hilbert module which will be the "nice modules" we will use for building blocks. This concept is a refinement of the notions of sharp kernel and kernel Hilbert space introduced by Curto and Salinas [13], Agrawal and Salinas [2].

Let $k, 1 \leq k \leq \infty$, be an arbitrary cardinal number and $\ell_{k}^{2}$ denote the $k$ - dimensional Hilbert space. Let $\mathcal{M}$ be a Hilbert module of rank $k$ over the algebra $\mathcal{A}(\Omega)$ relative to the generating set $\left\{f_{1}, f_{2}, \ldots\right\} \subseteq \mathcal{A}(\Omega)$ for which $\left\{f_{i} \otimes_{\mathcal{A}} 1_{\underline{z}}: 1 \leq i \leq k\right\}$ is linearly independent for $\underline{z} \in \Omega$. Since module multiplication by a function $\varphi$ on the module tensor product $\mathcal{M} \otimes_{\mathcal{A}(\Omega)} \mathbb{C}_{\underline{z}}$ is just multiplication by $\varphi(\underline{z})$, we see it must be isomorphic to the Hilbert space tensor product $\mathbb{C}_{\underline{z}} \otimes \ell_{k}^{2}$. Define the map $\Gamma: \mathcal{A}(\Omega) \otimes_{\text {alg }} \ell_{k}^{2} \rightarrow \mathcal{M}$ by $\Gamma\left(\sum \varphi_{i} \otimes e_{i}\right)=\sum \varphi_{i} f_{i}$, where $\left\{e_{i}\right\}_{i=1}^{k}$ is the standard orthonormal basis in $\ell_{k}^{2}$. We claim that $\Gamma$ is well defined, oneto-one and has dense range. Given the uniqueness of expressing an element $\phi=\sum \varphi_{i} \otimes e_{i}$ as a finite sum, we have that $\Gamma$ is well defined. If $\Gamma(\phi)=0$, then we have for $\underline{z} \in \Omega$ that $\sum \varphi_{i}(\underline{z})\left(f_{i} \otimes_{\mathcal{A}} 1_{\underline{z}}\right)=\left(\sum \varphi_{i} f_{i}\right) \otimes_{\mathcal{A}} 1_{\underline{z}}=\Gamma(\phi) \otimes_{\mathcal{A}} 1_{\underline{z}}=0$. Since the $f_{i}$ are linearly independent,

\footnotetext{
${ }^{1}$ In 6 , Arveson reaches the same conclusion, but he shows that dilations of a different nature seem to work well in his context.
} 
it follows that each $\varphi_{i}=0$ and hence $\phi=0$. Finally, the range of $\Gamma$ is dense since the $f_{i}$ 's form a generating set for $\mathcal{M}$. Now define the inner product $\langle$,$\rangle on \mathcal{A}(\Omega) \otimes_{\text {alg }} \ell_{k}^{2}$ such that $\langle\phi, \psi\rangle=\langle\Gamma \phi, \Gamma \psi\rangle_{\mathcal{M}}$. We complete $\mathcal{A}(\Omega) \otimes_{\text {alg }} \ell_{k}^{2}$ using this inner product to obtain a Hilbert module isometrically isomorphic to $\mathcal{M}$. Let $e_{\underline{z}}: \mathcal{A}(\Omega) \otimes_{\text {alg }} \ell_{k}^{2} \rightarrow \ell_{k}^{2}$ be the evaluation map at $\underline{z} \in \Omega$.

Let $X_{\underline{z}}: \mathcal{M} \otimes_{\mathcal{A}} \mathbb{C}_{\underline{z}} \rightarrow \mathbb{C}_{\underline{z}} \otimes \ell_{k}^{2}$ be the map defined by $X_{\underline{z}}\left(f_{i} \otimes_{\mathcal{A}} 1_{\underline{z}}\right)=1_{\underline{z}} \otimes e_{i}$, and extend linearly to finite linear sums.

LEMMA 1. The map $X_{\underline{z}}$ is bounded if the evaluation map $e_{\underline{z}}$ is bounded. Furthermore, $\left\|X_{\underline{z}}\right\|=\left\|e_{\underline{z}}\right\|$, for $\underline{z} \in \Omega$.

Proof. Let $\phi=\sum \varphi_{i} e_{i}$ be any arbitrary element of $\mathcal{A}(\Omega) \otimes_{\text {alg }} \ell_{k}^{2}$. In the following, $\|\phi\|_{\mathcal{M}}$ denotes the norm induced on $\mathcal{A}(\Omega) \otimes_{\text {alg }} \ell_{k}^{2}$ via the map $\Gamma$. First, let us compute the norm of the operator $e_{\underline{z}}: \mathcal{A}(\Omega) \otimes_{\text {alg }} \ell_{k}^{2} \rightarrow \ell_{k}^{2}$ as follows:

$$
\begin{aligned}
\left\|e_{\underline{z}}\right\| & =\sup _{\phi}\|\phi(\underline{z})\|_{\ell_{k}^{2}} /\|\phi\|_{\mathcal{M}} \\
& =\sup _{\phi}\left\|\sum \varphi_{i}(\underline{z}) e_{i}\right\|_{\ell_{k}^{2}} /\|\Gamma \phi\|_{\mathcal{M}} \\
& =\sup _{\phi}\left\|\sum \varphi_{i}(\underline{z}) e_{i}\right\|_{\ell_{k}^{2}} /\left\|\sum \varphi_{i} f_{i}\right\|_{\mathcal{M}} \\
& =\sup _{\phi, \Psi}\left\|\sum \varphi_{i}(\underline{z}) e_{i}\right\|_{\ell_{k}^{2}} /\left\|\sum\left(\varphi_{i}+\psi_{i}\right) f_{i}\right\|_{\mathcal{M}} \\
& =\sup _{\phi}\left\|\sum \varphi_{i}(\underline{z}) e_{i}\right\|_{\ell_{k}^{2}} / \inf _{\Psi}\left\|\sum\left(\varphi_{i}+\psi_{i}\right) f_{i}\right\|_{\mathcal{M}},
\end{aligned}
$$

where $\Psi=\left\{\psi_{1}, \ldots, \psi_{n}\right\}$ is a set of finitely many non-zero elements in $\mathcal{A}(\Omega)$ that vanish at $\underline{z} \in \Omega$. Note that, $\sum_{1 \leq i \leq n} \psi_{i} f_{i}$ is a collection of elements dense in $\mathcal{A}_{\underline{z}} \mathcal{M}$, where $\mathcal{A}_{\underline{z}}$ is the ideal of functions in $\mathcal{A}(\Omega)$ that vanish at $\underline{z}$. Therefore, we see that

$$
\inf _{\Psi}\left\|\sum\left(\varphi_{i}+\psi_{i}\right) f_{i}\right\|_{\mathcal{M}}=\left\|\left(\sum \varphi_{i} f_{i}\right) \otimes_{\mathcal{A}} 1_{\underline{z}}\right\|_{\mathcal{M} \otimes \mathcal{A} \mathbb{C} \underline{z}}
$$

Consequently, $\left\|e_{\underline{z}}\right\|=\sup \left\|\sum_{\phi} \varphi_{i}(\underline{z}) e_{i}\right\|_{\ell_{k}^{2}} /\left\|\left(\sum \varphi_{i} f_{i}\right) \otimes_{\mathcal{A}} 1_{\underline{z}}\right\|_{\mathcal{M} \otimes_{\mathcal{A}} \mathbb{C} \underline{z}}$. Since $\left\|\sum \varphi_{i}(\underline{z}) e_{i}\right\|_{\ell_{k}^{2}}=$ $\left\|\left(\sum \varphi_{i}(\underline{z}) e_{i}\right) \otimes 1_{\underline{z}}\right\|_{\ell_{k}^{2} \otimes \mathbb{C}_{\underline{z}}}$ is by definition the norm of the operator $X_{\underline{z}}$, it follows that $\left\|e_{\underline{z}}\right\|=$ $\left\|X_{\underline{z}}\right\|$ as claimed.

This Lemma prompts the following Definition.

Definition 1. A Hilbert module $\mathcal{R}$ over $\mathcal{A}(\Omega)$ is said to be quasi-free of rank $k$ relative to the generating set $\left\{f_{1}, f_{2}, \ldots\right\}$ for $1 \leq k \leq \infty$, if

(i) $f_{1} \otimes_{\mathcal{A}} 1_{\underline{z}}, f_{2} \otimes_{\mathcal{A}} 1_{\underline{z}}, \ldots$ forms a basis for the fiber $\operatorname{Sp}_{\underline{z}}(\mathcal{R})$ for $\underline{z} \in \Omega$,

(ii) the map $X_{\underline{z}}$ is locally uniformly bounded in norm, and

(iii) for $f$ in $\mathcal{R}, f \otimes_{\mathcal{A}} 1_{\underline{z}}=0$ for every $\underline{z} \in \Omega$ if and only if $f=0$ in $\mathcal{R}$.

When $k$ is finite, the combination of the requirements that $\operatorname{Sp}_{\underline{z}}(\mathcal{R})$ is $k$-dimensional and the localization of the generating set has cardinality $k$ has strong implications. For $k=\infty$, there are many different ways in which a set can be a basis. Clearly, we don't want to assume the set forms an orthonormal or even an orthogonal basis. But we may want to assume that the set of vectors $\left\{f_{1} \otimes_{\mathcal{A}} 1_{\underline{z}}, f_{2} \otimes_{\mathcal{A}} 1_{\underline{z}}, \cdots\right\}$ forms a basis in $\operatorname{Sp}_{\underline{z}}(\mathcal{R})$ equivalent to the standard basis. However, in this paper we assume only that the set is linearly independent and spans. 
There is another description which demonstrates the sense in which a quasi-free Hilbert module is "almost free". In commutative algebra, the statement that a module is free means that it is isomorphic to a direct sum of copies of the ring which in our case would be $\mathcal{A}(\Omega)$. But this can't happen, since the direct sum of copies of $\mathcal{A}(\Omega)$ can't be isomorphic to a Hilbert space unless $\mathcal{A}(\Omega)=\mathbb{C}$ which is impossible. Hence, we do the next best thing. Since we are interested in modules with a Hilbert space structure, we will begin with the "free module" $\mathcal{A}(\Omega) \otimes_{\text {alg }} \ell_{k}^{2}$ which is the algebraic tensor product of $\mathcal{A}(\Omega)$ with the Hilbert space $\ell_{k}^{2}, 1 \leq k \leq \infty$, and then complete it to obtain a Hilbert space.

A module $\mathcal{R}$ over $\mathcal{A}(\Omega)$, quasi-free relative to $\left\{f_{1}, f_{2}, \ldots\right\}$, is the Hilbert space completion of the free module $\mathcal{A}(\Omega) \otimes_{\text {alg }} \ell_{k}^{2}$ via the map $\Gamma$. Moreover, the following statement is an abstract characterization of such completions of $\mathcal{A}(\Omega) \otimes_{\mathrm{alg}} \ell_{k}^{2}$. The technique used in its proof is closely related to the proof of [24, Theorem 5.14].

Proposition 1. A Hilbert module $\mathcal{R}$ for $\mathcal{A}(\Omega)$ is quasi-free of rank $k$ for $1 \leq k \leq \infty$, relative to a generating set $\left\{f_{1}, f_{2}, \ldots\right\}$ if and only if it is isometrically isomorphic to the completion of $\mathcal{A}(\Omega) \otimes_{\mathrm{alg}} \ell_{k}^{2}$ with respect to a norm associated with an inner product such that

(a) evaluation of functions in $\mathcal{A}(\Omega) \otimes_{\mathrm{alg}} \ell_{k}^{2}$ at each point $\underline{z}$ in $\Omega$ is locally uniformly bounded,

(b) module multiplication on $\mathcal{A}(\Omega) \otimes_{\text {alg }} \ell_{k}^{2}$ by functions in $\mathcal{A}(\Omega)$ is continuous, and

(c) for $\left\{\phi_{n}\right\}$ contained in $\mathcal{A}(\Omega) \otimes_{\text {alg }} \ell_{k}^{2}$ which is Cauchy in norm, we have $\left\|\phi_{n}(\underline{z})\right\|_{\ell_{k}^{2}} \rightarrow 0$ for all $\underline{z} \in \Omega$ if and only if $\left\|\phi_{n}\right\| \rightarrow 0$.

Proof. We first show that the inner product introduced on $\mathcal{A}(\Omega) \otimes \ell_{k}^{2}$ using the map $\Gamma$ satisfies conditions (a), (b) and (c). Let $\mathcal{R}$ be a quasi-free module. Then the local uniform boundedness of the map $X_{\underline{z}}$ together with the equality $\left\|X_{\underline{z}}\right\|=\left\|e_{\underline{z}}\right\|$, for $\underline{z} \in \Omega$ establishes the condition (a). For the proof of (b), consider the function $\psi$ in $\mathcal{A}(\Omega)$ and observe that $\left\|\psi \sum \varphi_{i} \otimes e_{i}\right\|=\left\|\sum \psi \varphi_{i} \otimes e_{i}\right\|=\left\|\Gamma\left(\sum \psi \varphi_{i} \otimes e_{i}\right)\right\|_{\mathcal{R}}=\left\|\psi \Gamma\left(\sum \varphi_{i} \otimes e_{i}\right)\right\|_{\mathcal{R}} \leq C_{1}\|\psi\| \| \Gamma\left(\sum \varphi \otimes\right.$ $\left.e_{i}\right)\left\|_{\mathcal{R}} \leq C_{1}\right\| \psi\|\| \sum\left(\varphi_{i} \otimes e_{i}\right) \|$. Finally, let $\phi_{n}=\sum \varphi_{i}^{(n)} \otimes e_{i}$ be a sequence in $\mathcal{A}(\Omega) \otimes_{\text {alg }} \ell_{k}^{2}$, which is Cauchy in norm. Then $\Gamma\left(\phi_{n}\right) \rightarrow g$ for some $g \in \mathcal{R}$. Since $\Gamma$ is continuous, it follows that $e_{\underline{z}}\left(\phi_{n}\right) \rightarrow 0$ if and only if $e_{\underline{z}}\left(\Gamma\left(\phi_{n}\right)\right) \rightarrow 0$. Or, in other words, $\phi_{n}(\underline{z}) \rightarrow 0$ if and only if $\sum \varphi_{n}(\underline{z}) f_{i} \otimes_{\mathcal{A}} 1_{\underline{z}} \rightarrow 0$. Hence, the assumption that $\phi_{n}(\underline{z}) \rightarrow 0$ implies that $g(\underline{z})=0$ and hence $g=0$ by (iii) of Definition 1. This shows that the condition (c) holds which completes the proof in the first direction.

For the converse, assume that $\mathcal{R}$ is the completion of $\mathcal{A}(\Omega) \otimes_{\text {alg }} \ell_{k}^{2}$ with respect to an inner product that satisfies (a), (b), and (c) of the Proposition. We must verify that the conditions of Defintion 1 hold. Fix $\underline{z} \in \Omega$ and consider the map $F_{\underline{z}}: \mathcal{A}(\Omega) \otimes_{\text {alg }} \ell_{k}^{2} \rightarrow \ell_{k}^{2}$ defined by $F_{z}\left(\sum \varphi_{i} \otimes e_{i}\right)=\sum \varphi_{i}(\underline{z}) e_{i}$. By condition (a) of the Proposition, it follows that $F$ extends to a map from $\mathcal{R}$ to $\ell_{k}^{2}$. We use $F_{\underline{z}}$ to define a map $F_{\underline{z}}^{\prime}: \mathcal{R} \otimes \mathbb{C}_{\underline{z}} \rightarrow \mathbb{C}_{\underline{z}} \otimes \ell_{k}^{2}$ such that $F_{\underline{z}}^{\prime}\left(\left(\sum \varphi_{i} \otimes e_{i}\right) \otimes a=\sum \varphi_{i}(\underline{z}) e_{i} \otimes a\right.$. The kernel of this map is spanned by the vectors $\varphi \otimes e_{i} \otimes a-1 \otimes e_{i} \otimes \varphi(\underline{z}) a$ for $\varphi_{i} \in \mathcal{A}(\Omega)$ and $a \in \mathbb{C}_{\underline{z}}$, which is the submodule used to define the module tensor product $\mathcal{R} \otimes_{\mathcal{A}} \mathbb{C}_{\underline{z}}$. Hence, we see that evaluation of $\sum \varphi_{i} \otimes e_{i}$ at $\underline{z}$ matches $\sum \varphi_{i}(\underline{z}) e_{i}$ in $\mathbb{C}_{\underline{z}} \otimes \ell_{k}^{2}$ which establishes (i) in Definition 11. The condition (ii) of the Definition is clearly the same as condition (a) of the Proposition. Also, this matchup shows that condition (c) of the proposition implies (iii) of Defintiion 1, which completes the proof. 
Observe that no assumption of holomorphicity is made in the definition of a quasi-free Hilbert module $\mathcal{R}$. However, identification of $\mathcal{R}$ with the completion of $\mathcal{A}(\Omega) \otimes_{\text {alg }} \ell_{k}^{2}$ in the finite rank case makes the spectral sheaf $\operatorname{Sp}(\mathcal{R})$ into a hermitian holomorphic vector bundle of rank $k$ with holomorphic frame $\underline{z} \rightarrow\left\{f_{i} \otimes_{\mathcal{A}} 1_{\underline{z}}: 1 \leq i \leq k\right\}$. Moreover, using the identification of $\mathcal{R}$ with the completion of $\mathcal{A}(\Omega) \otimes_{\text {alg }} \ell_{k}^{2}$, we see that $\mathcal{R}$ can be realized as a space of $\ell_{k}^{2}$ - valued holomorphic functions on $\Omega$ which forms a kernel Hilbert space.

Obviously, the Hardy and Bergman modules are quasi-free. While submodules of quasifree modules are not quasi-free in general, principal submodules are, since one can view them as being obtained merely from a change of norm. Quotient modules of quasi-free Hilbert modules are seldom quasi-free. However, it can happen. The relationship here is analogous to the situation of holomorphic subbundles of holomorphic bundles; sometimes there is a holomorphic complement. The following statement should be true in our context and would clarify the relation between the notions of free and projective.

Conjecture 1. Let $\mathcal{R}$ be a quasi-free Hilbert module of rank $k, 1 \leq k<\infty$, over $\mathcal{A}(\Omega)$ and $\mathcal{R}_{1}$ and $\mathcal{R}_{2}$ be submodules of $\mathcal{R}$ such that $\mathcal{R}$ is the algebraic direct sum of $\mathcal{R}_{1}$ and $\mathcal{R}_{2}$. Then $\mathcal{R}_{1}$ and $\mathcal{R}_{2}$ are quasi-free of ranks $k_{1}$ and $k_{2}$, respectively, and $k=k_{1}+k_{2}$.

Something analogous should be true in the case $k=\infty$ but would probably require more explicit hypotheses on the angle between the two submodules.

\section{Regular Modules}

As indicated earlier, a resolution of the Hilbert module $\mathcal{M}$ is a sequence of modules $\left\{\mathcal{R}_{i}\right\}$, either of finite or infinite length, with module map $X_{0}: \mathcal{R}_{1} \rightarrow \mathcal{M}$ and module maps $X_{i}: \mathcal{R}_{i+1} \rightarrow \mathcal{R}_{i}$ for $i \geq 1$, such that the sequence

$$
0 \longleftarrow \mathcal{M} \stackrel{X_{0}}{\longleftarrow} \mathcal{R}_{1} \stackrel{X_{1}}{\longleftarrow} \mathcal{R}_{2} \stackrel{X_{2}}{\longleftarrow} \cdots
$$

is exact in the sense that $X_{0}$ is onto and $\operatorname{ker} X_{i}=\operatorname{ran} X_{i+1}$ for $i \geq 1$. If the sequence is of finite length, then we must have the final $\mathcal{R}_{i}=0$. One speaks of a weak resolution if it is only topologically exact or, equivalently, if one assumes that $X_{0}$ has dense range and ker $X_{i}=$ clos $\operatorname{ran} X_{i+1}$ for $i \geq 1$. We are assuming in all cases that the modules $\left\{\mathcal{R}_{i}\right\}$ are quasi-free over $\mathcal{A}(\Omega)$. One can also put an additional restriction on the module maps by requiring that they are partial isometries in which case we will speak of a strong resolution.

The resolution obtained from the Sz.-Nagy-Foias model is a strong resolution, while the second one constructed for the Hilbert module $\mathbb{C}_{\underline{0}}$ over $\mathcal{A}\left(\mathbb{D}^{2}\right)$ is a resolution but not a strong one. Although one seeks resolutions as nice as possible, and closely related to $\mathcal{M}$, one often faces tradeoffs. For example, the inclusion map of the Hardy module $H^{2}(\mathbb{D})$ into the Bergman module $B^{2}(\mathbb{D})$ defines a weak resolution with just one term since the map has dense range and trivial kernel. However, it is not clear just what this resolution can tell us about the Bergman module in terms of the Hardy module. On the other hand, while the resolution of the Bergman module given by the Sz.-Nagy-Foias model is a strong one, it is obtained at the price of having to deal with Hardy modules of infinite multiplicity. Still we show in section 5 that all resolutions, even weak ones, contain information about the module. Finally, in that section we will also compare the existence questions for the various kinds of resolutions. But first we want to investigate existence in general.

If one is given a Hilbert module $\mathcal{M}$, the first task in constructing a resolution of $\mathcal{M}$ by quasi-free Hilbert modules is to obtain a quasi-free Hilbert module $\mathcal{R}$ and module map 
$X: \mathcal{R} \rightarrow \mathcal{M}$ with dense range. We introduce the following definitions to capture the kinds of behavior possible with regard to the construction of resolutions.

Definition 2. A Hilbert module over $\mathcal{A}(\Omega)$ is said to be

(1) weakly regular if there exists a quasi-free Hilbert module $\mathcal{R}$ over $\mathcal{A}(\Omega)$ and a module map $X: \mathcal{R} \rightarrow \mathcal{M}$ with dense range,

(2) regular if the map $X$ can be taken to be onto,

(3) strongly regular if the map $X$ can be taken to be a co-isometry, and

(4) singular if the only module map $X: \mathcal{R} \rightarrow \mathcal{M}$ from a quasi-free Hilbert module $\mathcal{R}$ to $\mathcal{M}$ is the zero map.

It is relatively straight forward to see that not all Hilbert modules are weakly regular. In particular, we will see that for the Hilbert module $\mathbb{C}_{1}$ over $\mathcal{A}(\mathbb{D})$, the only module map $X: \mathcal{R} \rightarrow \mathbb{C}_{1}$ for $\mathcal{R}$ a quasi-free Hilbert module, is the zero map. Toward that end, we recall an extension of a notion of Sz.-Nagy and Foias 33] to the context of Hilbert modules (cf. [1]).

Definition 3. A Hilbert module $\mathcal{M}$ over $\mathcal{A}(\Omega)$ is said to belong to class $C_{0}$ if for every sequence $\left\{\varphi_{n}\right\}_{n \in \mathbb{N}}$ in the unit ball of $\mathcal{A}(\Omega)$ satisfying $\varphi_{n}(\underline{z}) \rightarrow 0$ for $\underline{z}$ in $\Omega$ it follow that $M_{\varphi_{n}}^{*} \rightarrow 0$ in the strong operator topology.

One could also assume that the $\varphi_{n}$ converge uniformly to zero on compact subsets of $\Omega$. In many situations, these two notions seem to coincide but it is not clear if they do for general Hilbert modules.

Proposition 2. A regular Hilbert module over $\mathcal{A}(\Omega)$ belongs to class $C_{.0}$.

Proof. Let $\mathcal{M}$ be a regular Hilbert module with $\mathcal{R}$ a quasi-free Hilbert module and $X$ : $\mathcal{R} \rightarrow \mathcal{M}$ an onto module map. If $k_{z}$ in $\mathcal{R}$ is a common eigenvector for the adjoint of module multiplication on $\mathcal{R}$, then $M_{\varphi_{n}}^{*} k_{z}=\overline{\varphi_{n}(z)} k_{z}$ and hence $\left\|M_{\varphi_{n}}^{*} k_{z}\right\| \rightarrow 0$. Since the vectors $\left\{k_{z}\right\}, z \in \mathbb{D}$ span $\mathcal{R}$ and $\left\|M_{\varphi_{n}}^{*}\right\| \leq \alpha\left\|\varphi_{n}\right\| \leq \alpha$, it follows that $M_{\varphi_{n}}^{*} \rightarrow 0$ in the strong operator topology. Then, $X^{*} N_{\varphi}^{*}=M_{\varphi}^{*} X^{*}$, where $N_{\varphi}$ denotes the operator defined by module multiplication on $\mathcal{M}$. Since $X$ is onto, it follows that $X^{*}$ is bounded below and hence $N_{\varphi}^{*} \rightarrow 0$ in the strong operator topology. Thus $\mathcal{M}$ belongs to class $C_{\cdot 0}$.

Taking $X$ to be the identity map, this result yields a property of quasi-free Hilbert modules.

Corollary 1. All quasi-free Hilbert modules belong to class $C_{.0}$.

Proposition 3. The Hilbert module $\mathbb{C}_{1}$ over $\mathcal{A}(\mathbb{D})$ does not belong to class $\mathbb{C}_{.0}$.

Proof. There exists a sequence $\left\{\varphi_{n}\right\}_{n \in \mathbb{N}} \in \mathcal{A}(\mathbb{D})$ satisfying $\varphi_{n}(1)=1,\left\|\varphi_{n}\right\|=1$, and $\varphi_{n}(z) \rightarrow 0$ for $z \in \mathbb{D}$. For example, one can take $\varphi_{n}(z)=1 / n(1+1 / n-z)$. Then for $\lambda$ in $\mathbb{C}_{1}$ we see that $M_{\varphi_{n}}^{*} \lambda=\overline{\varphi_{n}(1)} \lambda=\lambda$ does not converge to zero which completes the proof.

We have been unable to show either that weakly regular implies class $C_{.0}$ or that class $C_{.0}$ implies weak regularity. However, the first conclusion would seem to be likely.

Although we have been unable to obtain an intrinsic characterization of weak regularity, we can provide two properties each of which implies it, both relating to the notion that the module is supported on the interior of $\Omega$. 
Definition 4. Let $\mathcal{R}$ be a quasi-free Hilbert module of rank one over $\mathcal{A}(\Omega)$ for the generating vector $g$ and let $\mathcal{M}$ be a Hilbert module over $\mathcal{A}(\Omega)$. Then $\mathcal{M}$ is said to be smooth relative to $\mathcal{R}$ and $g$ if the map $S: \mathcal{M} \rightarrow \mathcal{M} \otimes_{\mathcal{A}(\Omega)} \mathcal{R}$ defined by $S f=f \otimes_{\mathcal{A}(\Omega)} g$ is one-to-one.

Smooth modules in this sense are not always in class $C_{.0}$. Consider the contractive Hilbert module over $\mathcal{A}(\mathbb{D})$ defined by a function $\theta$ in $H^{\infty}(\mathbb{D})$ with $\left|\theta\left(e^{i t}\right)\right|<1$ on a set of positive measure. It has the form $\mathcal{H}=H^{2}(\mathbb{D}) \oplus L^{2}(\bar{\Delta}) /\left\{\theta f \oplus \Delta f: f \in H^{2}(\mathbb{D})\right\}$, where $\Delta\left(e^{i t}\right)=$ $\left(1-\left|\theta\left(e^{i t}\right)\right|^{2}\right)^{1 / 2}$ and $\bar{\Delta}$ is the characteristic function for the support of $\Delta$. The map $S$ : $H^{2}(\mathbb{D}) \oplus L^{2}(\bar{\Delta} \mathbb{T}) \rightarrow\left(H^{2}(\mathbb{D}) \oplus L^{2}(\bar{\Delta} \mathbb{T})\right) \otimes_{\mathcal{A}(\mathbb{D})} H^{2}(\mathbb{D})$ reduces to $S(f \oplus g)=f \otimes_{\mathcal{A}(\mathbb{D})} 1$ since $L^{2}(\mathbb{D}) \otimes_{\mathcal{A}(\mathbb{D})} H^{2}(\mathbb{D})=\{0\}$. Since the map from $H^{2}(\mathbb{D})$ to $H^{2}(\mathbb{D}) \otimes_{\mathcal{A}(\mathbb{D})} H^{2}(\mathbb{D})$ is one-to-one, we see that $S(h \oplus k)=0$ implies $h=0$. For such a vector to be in $\mathcal{H}$, it must be orthogonal to the subspace $\left\{\theta f \oplus \Delta f: f \in H^{2}(\mathbb{D})\right\}$. We can choose $\theta$ so that this subspace is dense in $L^{2}(\bar{\Delta} \mathbb{T})$ in which case $\mathcal{H}$ is $H^{2}(\mathbb{D})$-smooth. One can show that such a Hilbert module does not belong to the class $C_{.0}$.

In general, the question of whether $\mathcal{H}$ is $H^{2}(\mathbb{D})$-smooth depends on the density of $\{\Delta f\}$ in $L^{2}(\bar{\Delta} \mathbb{T})$ and that happens when the associated contraction on $\mathcal{H}$ has no co-isometric part. That relationship seems to hold for general contractive Hilbert modules although a precise result would depend on an analysis of how the notion of smoothness depends on the quasifree Hilbert module $\mathcal{R}$ and the generating vector used to define the $S$-map. In particular, although we presume the class to be independent of both $\mathcal{R}$ and $g$, we have been unable to prove that.

Definition 5. A Hilbert module is said to belong to the class (SM) if it is smooth for some quasi-free Hilbert module $\mathcal{R}$ and generating vector $g$.

Although the notion of smoothness is conceptually appealing, it is not always easy to verify. We introduce a subclass, whose membership is more closely related to operator theoretic ideas which we recall first.

Definition 6. If $\mathcal{M}$ is a Hilbert module over $\mathcal{A}(\Omega)$, then a vector $h \in \mathcal{M}$ is said to be a common generalized eigenvector for the adjoint of module multiplication if $h$ lies in the kernel of $\left(M_{\varphi}-\varphi(\underline{z}) I\right)^{* n}$ for all $\varphi$ in $\mathcal{A}(\Omega)$ and some fixed positive integer $n$.

The subclass of the class (SM) we want to define involves the assumption that common generalized eigenvectors span.

Definition 7. A Hilbert module is said to belong to the class (PS) if $\mathcal{M}$ is spanned by the generalized eigenvectors for the adjoint of module multiplication.

Another characterization of class (PS) is possible using the notion of higher order localization. We will not discuss this notion in complete detail here but see [21]. Consider a point $\underline{z}$ in $\Omega$ and let $\mathcal{I}_{\underline{z}}$ be the ideal of polynomials in $\mathbb{C}[\underline{z}]$ that vanish at $\underline{z}$ and $\mathcal{I}_{\underline{z}}^{n}$ the ideal generated by the products of $n$ elements in $\mathcal{I}_{\underline{z}}$. The quotient $\mathcal{Q}_{\underline{z}}^{n}=\mathbb{C}[\underline{z}] / \mathcal{I}_{\underline{z}}^{n}$ with the Hilbert space structure in which the set of mononials in the quotient form an orthonormal basis, is a Hilbert module over $\mathcal{A}(\Omega)$ of dimension $m n$ in which module multiplication by a function $\varphi$ depends on the values at $\underline{z}$ of $\varphi$ and its partial derivatives of order less than $n$. Let $e$ denote the element of $\mathcal{Q}_{\underline{z}}^{n}$ corresponding to the monomial 1. Using elementary calculations, one can show that the following proposition provides another characterization of the class (PS). 
Proposition 4. A Hilbert module $\mathcal{M}$ over $\mathcal{A}(\Omega)$ belongs to class (PS) if and only if for every nonzero vector $f \in \mathcal{M}$, there exists $\underline{z} \in \Omega$ and $n$ such that the image $f \otimes_{\mathcal{A}} e$ in $\mathcal{M} \otimes_{\mathcal{A}} \mathcal{Q}_{\underline{z}}^{n}$ of $f$ is not 0 . Equivalently, the intersection of the closures of the submodules $M\left(\underline{z}_{1}, n_{1} ; \underline{z}_{2}, n_{2} ; \ldots ; \underline{z}_{k}, n_{k}\right)$ is $\{0\}$, where $M\left(\underline{z}_{1}, n_{1} ; \ldots ; \underline{z}_{k}, n_{k}\right)$ is the closure of the product $\mathcal{I}_{\underline{z}_{1}}^{n_{1}} \ldots \mathcal{I}_{\underline{z}_{k}}^{n_{k}}$ acting on $\mathcal{M}$ for every finite subset $\underline{z}_{1}, \ldots, \underline{z}_{k}$ of $\Omega$ and positive integers $n_{1}, \ldots, n_{k}$.

Proof. The equivalence of the two statements is an easy exercise involving the relation of the submodule which defines the tensor product of $\mathcal{M}$ with $\mathcal{Q}_{\underline{z}}^{n}$ and the closure of the range of $\mathcal{I}_{\underline{z}}$ acting on $\mathcal{M}$ (cf. [24, Theorem 5.14]). Similarly, by considering the relation of the kernel of the adjoint action of the nth power of a function $\varphi$ which vanishes at $\underline{z}$ and the latter space, one shows the equivalence with $\mathcal{M}$ belonging to class (PS).

It is not true that the modules belonging to class (PS) are just those determined by their spectral sheaves which are, of course, in class (PS); the higher multiplicity examples in [21] show otherwise. However, quasi-free Hilbert modules are determined by their spectral sheaves and there are relationships between the classes $C_{.0}$ and (PS).

Corollary 2. A quasi-free Hilbert module for $\mathcal{A}(\Omega)$ belongs to class (PS).

Proposition 5. A Hilbert module in class (PS) belongs to class C.0.

The proof is essentially the same as that of Proposition 2. The converse to the proposition is false, that is, Hilbert modules of class $C_{.0}$ do not necessarily belong to class (PS). For example, let $\theta$ be a singular inner function on the unit disk and $\mathcal{M}$ be the quotient Hilbert module $H^{2}(\mathbb{D}) / \theta H^{2}(\mathbb{D})$. Then the quotient map from the quasi-free Hilbert module $H^{2}(\mathbb{D})$ onto $\mathcal{M}$ shows that it is $C_{.0}$ but there are no common eigenvectors in $\mathbb{D}$ for the adjoint of module multiplication since the spectrum of $\mathcal{M}$ is the closed support of the singular measure that defines $\theta$ and hence a subset of $\partial \mathbb{D}$.

Proposition 6. A Hilbert module in class (PS) is in class (SM).

Proof. Let $\mathcal{M}$ be in class (PS) and let $\mathcal{R}$ be a quasi-free Hilbert module of rank one over $\mathcal{A}(\Omega)$ with generating vector $g$ and $S$ be the map $S: \mathcal{M} \rightarrow \mathcal{M} \otimes_{\mathcal{A}(\Omega)} \mathcal{R}$ defined by $S k=k \otimes_{\mathcal{A}(\Omega)} g$ for $k \in \mathcal{M}$. For $f$ a vector in $\mathcal{M}$, there exists a point $\underline{z} \in \Omega$ and an integer $n$ such that $f \otimes_{\mathcal{A}(\Omega)} e \neq 0$ in $\mathcal{M} \otimes_{\mathcal{A}(\Omega)} \mathcal{Q}$, where the module $\mathcal{Q}$ stands for $\mathcal{Q}_{z}^{n}$ and the $e$ as defined above. Let $X$ be the map from $\mathcal{M}$ to $\mathcal{M} \otimes_{\mathcal{A}(\Omega)} \mathcal{Q}$ defined by $X h=h \otimes_{\mathcal{A}(\Omega)} e$ and consider the diagram

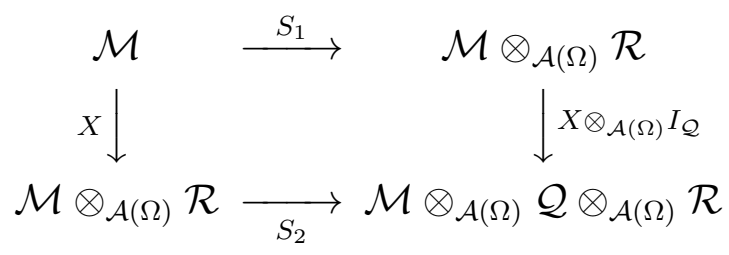

Then, $\left(X \otimes_{\mathcal{A}(\Omega)} I_{\mathcal{Q}}\right) S_{1} f=S_{2} X f=S_{2}\left(f \otimes_{\mathcal{A}(\Omega)} e\right) \neq 0$ since $\mathcal{Q} \rightarrow \mathcal{Q} \otimes_{\mathcal{A}(\Omega)} \mathcal{R}$ is an isomorphism. Hence, $S_{1} f \neq 0$ and $\mathcal{M}$ is smooth for $\mathcal{R}$ and $g$ which completes the proof.

Proposition 7. If $\mathcal{M}$ is a Hilbert module over $\mathcal{A}(\Omega)$ and $\mathcal{M}_{0}$ is a submodule of $\mathcal{M}$, then $\mathcal{M}$ in class $C_{.0}$ implies $\mathcal{M}_{0}$ is in class $C_{.0}$ and $\mathcal{M}$ in class (PS) implies $\mathcal{M}_{0}$ is in class (PS) and $\mathcal{M}$ in class $(\mathrm{SM})$ implies $\mathcal{M}_{0}$ is in class $(\mathrm{SM})$.

These results follow using similar arguments as before. 
Corollary 3. A submodule of a quasi-free Hilbert module over $\mathcal{A}(\Omega)$ belongs to classes $C_{.0},(\mathrm{PS})$ and $(\mathrm{SM})$.

We show in the next section that Hilbert modules of class (PS) and (SM) are weakly regular. With this result and the above corollary which we can use to show that every weakly regular Hilbert module has a weak resolution, we will conclude that Hilbert modules in class (PS) and (SM) have weak resolutions.

There is another class of Hilbert modules, which includes those in class (PS), for which we can also establish the existence of weak resolutions.

Definition 8. A Hilbert module $\mathcal{M}$ over $\mathcal{A}(\Omega)$ is said to be compactly supported on a vector $f$ in $\mathcal{M}$ if there is a compact subset $X$ of $\Omega$ and a constant $\beta$ such that

$$
\|\varphi f\|_{\mathcal{M}} \leq \beta\|\varphi\|_{X}\|f\|_{\mathcal{M}} \text { for } \varphi \in \mathcal{A}(\Omega),
$$

where $\|\varphi\|_{X}$ denotes the supremum norm of $\varphi$ taken on $X$. The module $\mathcal{M}$ is said to be compactly supported if there is a compact set $X$ and a constant $\beta$ which works for all vectors $f$ in $\mathcal{M}$. Finally, the module $\mathcal{M}$ is said to be almost compactly supported if $\mathcal{M}$ is the span of the compactly supported vectors in $\mathcal{M}$, where the compact set and constant can depend on a vector.

\section{Construction of Resolution}

We now introduce our basic construction for establishing weak regularity. Let $\mathcal{M}$ be a Hilbert module over $\mathcal{A}(\Omega)$ with a set of $k$ generators $\left\{f_{i} \in \mathcal{M}: 1 \leq i \leq k\right\}$, and let $\mathcal{R}$ be any quasi-free rank $k$ Hilbert module over $\mathcal{A}(\Omega)$ relative to $\left\{g_{i} \in \mathcal{R}: 1 \leq i \leq k\right\}, 1 \leq k \leq \infty$. Let $\mathcal{R}_{\mathcal{M}}$ be the closed submodule of $\mathcal{M} \oplus \mathcal{R}$ spanned by the vectors

$$
\left\{\varphi_{i} f_{i} \oplus \varphi_{i} g_{i}: \varphi_{i} \in \mathcal{A}(\Omega), 1 \leq i \leq k\right\}
$$

and let $P$ and $Q$ be the module maps from $\mathcal{R}_{\mathcal{M}}$ to $\mathcal{M}$ and $\mathcal{R}$, respectively, defined by $P\left(\varphi_{i} f_{i} \oplus \varphi_{i} g_{i}\right)=\varphi_{i} f_{i}$ and $Q\left(\varphi_{i} f_{i} \oplus \varphi_{i} g_{i}\right)=\varphi_{i} g_{i}$, respectively. Since a dense set of vectors in $\mathcal{R}_{\mathcal{M}}$ has the form $\Phi=\sum \varphi_{i} f_{i} \oplus \varphi_{i} g_{i}$, where at most finitely many of the $\varphi_{i}$ are nonzero, we see that $\|P \Phi\|=\left\|\sum \varphi_{i} f_{i}\right\| \leq\left(\left\|\sum \varphi_{i} f_{i}\right\|^{2}+\left\|\sum \varphi_{i} g_{i}\right\|^{2}\right)^{1 / 2}=\|\Phi\|$ and similarly, $\|Q \Phi\| \leq\|\Phi\|$. Thus both $P$ and $Q$ are well-defined, contractive and have dense range. If $\mathcal{R}_{\mathcal{M}}$ is quasi-free, then it will follow that $\mathcal{M}$ is weakly regular.

Consider the operator $e_{\underline{z}}: \mathcal{A}(\Omega) \otimes_{\text {alg }} \ell_{k}^{2} \rightarrow \ell_{k}^{2}$ of evaluation at $\underline{z}$ in $\Omega$. For the function $\phi=\sum \varphi \otimes e_{i}$ in $\mathcal{A}(\Omega) \otimes_{\text {alg }} \ell_{k}^{2}$ we have $e_{\underline{z}}(\phi)=\sum \varphi_{i}(\underline{z}) e_{i}$, and hence

$$
\begin{aligned}
\left\|e_{\underline{z}}\right\| & =\sup \left\{\left\|e_{\underline{z}}(\phi)\right\| /\|\phi\|: \phi \in \mathcal{A}(\Omega) \otimes_{\text {alg }} \ell_{k}^{2}\right\}, \\
& =\sup \left\{\left\|\sum \varphi_{i}(\underline{z}) e_{i}\right\| /\|\phi\|: \phi \in \mathcal{A}(\Omega) \otimes_{\text {alg }} \ell_{k}^{2}\right\} .
\end{aligned}
$$

Now, consider the evaluation, first on $\mathcal{R}$ and then on $\mathcal{R}_{\mathcal{M}}$, at $\underline{z}$. Recall that $\mathcal{A}(\Omega) \otimes_{\text {alg }} \ell_{k}^{2}$ is a dense spanning set in both $\mathcal{R}$ and $\mathcal{R}_{\mathcal{M}}$. It is clear that

$$
\left\|e_{\underline{z}}\right\|_{\mathcal{R} \rightarrow \ell_{k}^{2}}=\sup \left\{\left\|\sum \varphi_{i}(\underline{z}) e_{i}\right\| /\|\phi\|: \phi \in \mathcal{A}(\Omega) \otimes_{\mathrm{alg}} \ell_{k}^{2}\right\} .
$$

However,

$$
\begin{aligned}
\left\|e_{\underline{z}}\right\|_{\mathcal{R}_{\mathcal{M}} \rightarrow \ell_{k}^{2}} & =\sup \left\{\left\|e_{\underline{z}}(\phi)\right\| /\|\Gamma(\phi)\|: \phi \in \mathcal{A}(\Omega) \otimes_{\text {alg }} \ell_{k}^{2}\right\}, \\
& =\sup \left\{\left\|\sum \varphi_{i}(\underline{z}) e_{i}\right\| /\left\|\sum \varphi_{i}\left(f_{i} \oplus\left(1 \otimes e_{i}\right)\right)\right\|: \phi \in \mathcal{A}(\Omega) \otimes_{\text {alg }} \ell_{k}^{2}\right\},
\end{aligned}
$$


where $\Gamma: \mathcal{A}(\Omega) \otimes_{\text {alg }} \ell_{k}^{2} \rightarrow \mathcal{R}_{\mathcal{M}}$ is the map defined by $\Gamma(\phi)=\sum \varphi_{i}\left(f_{i} \oplus\left(1 \otimes e_{i}\right)\right)$. So, it follows that the norm of the evaluation operator on $\mathcal{R}$ dominates that of $\mathcal{R}_{\mathcal{M}}$ at $\underline{z}$.

Lemma 2. The Hilbert module $\mathcal{R}_{\mathcal{M}}$ over $\mathcal{A}(\Omega)$ is quasi-free of rank $k$ relative to the generating set $\left\{f_{i} \oplus g_{i}\right\}$ if $\operatorname{ker} Q=\{0\}$.

Proof. We will establish the three properties for a module to be quasi-free given in Definition 1. First, the norm of the evaluation operator $\left\|e_{\underline{z}}\right\|_{\mathcal{R}_{\rightarrow} \ell_{k}^{2}}$ is locally uniformly bounded. Since $\left\|e_{\underline{z}}\right\|_{\mathcal{R}_{\mathcal{M}} \rightarrow \ell_{k}^{2}} \leq\left\|e_{\underline{z}}\right\|_{\mathcal{R}_{\rightarrow} \ell_{k}^{2}}$, it follows that the norm of the evaluation operator $\left\|e_{\underline{z}}\right\|_{\mathcal{R}_{\mathcal{M}}}$ is locally uniformly bounded as well, which establishes (ii).

Now, for $\underline{z}$ in $\Omega$ and $1 \leq i \leq k$, we consider the set $\left\{\left(f_{i} \oplus g_{i}\right) \otimes_{\mathcal{A}} 1_{\underline{z}}\right\}$ in $\mathcal{R}_{\mathcal{M}} \otimes_{\mathcal{A}} \mathbb{C}_{\underline{z}}$. Since the set $\left\{f_{i} \oplus g_{i}\right\}$ generates $\mathcal{R}_{\mathcal{M}}$, it follows that the set $\left(f_{i} \oplus g_{i}\right) \otimes_{\mathcal{A}} 1_{\underline{z}}$ generates $\mathcal{R}_{\mathcal{M}} \otimes_{\mathcal{A}} \mathbb{C}_{\underline{z}}$ as a module over $\mathcal{A}(\Omega)$. However, from the fact that $\mathcal{R}_{\mathcal{M}} \otimes_{\mathcal{A}} \mathbb{C}_{\underline{z}}$ is isomorphic to a direct sum of copies of $\mathbb{C}_{\underline{z}}$, it follows that a generating set over $\mathcal{A}(\Omega)$ is the same as a generating set over $\mathbb{C}$ or as a Hilbert space. Further, since the set of vectors $\left\{g_{i} \otimes_{\mathcal{A}} 1_{\underline{z}}\right\}$ is linearly independent in $\mathcal{R} \otimes_{\mathcal{A}} \mathbb{C}_{\underline{z}}$, it follows that the set $\left\{\left(f_{i} \oplus g_{i}\right) \otimes_{\mathcal{A}} 1_{\underline{z}}\right\}$ is linearly independent in $\mathcal{R}_{\mathcal{M}} \otimes_{\mathcal{A}} \mathbb{C}_{\underline{z}}$, which is condition (i).

Thus, whether $\mathcal{R}_{\mathcal{M}}$ is quasi-free comes down to whether or not condition (iii) holds. Suppose $h$ is a vector in $\mathcal{R}_{\mathcal{M}}$ such that $h \otimes_{\mathcal{A}} 1_{\underline{z}}=0$ for every $\underline{z} \in \Omega$. Then we have

$$
(Q h) \otimes_{\mathcal{A}} 1_{\underline{z}}=\left(Q \otimes_{\mathcal{A}} 1_{\underline{z}}\right)\left(h \otimes_{\mathcal{A}} 1_{\underline{z}}\right)=0
$$

and, since $Q h$ is in $\mathcal{R}$ which is quasi-free, we have $Q h=0$. If $\operatorname{ker} Q=\{0\}$, then $\mathcal{R}_{\mathcal{M}}$ is quasi-free which completes the proof.

A reasonable question that arises is whether $\operatorname{ker} Q=\{0\}$ always holds. To see that is not the case, consider $\mathcal{R}=H^{2}(\mathbb{D})$ and $\mathcal{M}=\mathbb{C}_{1}$ over $\mathcal{A}(\mathbb{D})$. One can either repeat the arguments from the last section or observe that if $\operatorname{ker} Q=\{0\}$ in this case, it would follow from the lemma and later results in this section that $\mathbb{C}_{1}$ is weakly regular. Since $\mathbb{C}_{1}$ is finite dimensional, we have that $\mathbb{C}_{1}$ is regular which would imply that $\mathbb{C}_{1}$ is in class $C_{.0}$ by Proposition 2, a contradiction. There is another conclusion one can draw from this example, namely that the third condition in the definition of quasi-free does not follow automatically from the first two. In particular, if one considers the function 1 as a generator for $H^{2}(\mathbb{D})$ and the scalar 1 as a generator for $\mathbb{C}_{1}$, the $\mathcal{R}_{\mathcal{M}}$ space in this case is $H^{2}(D) \oplus \mathbb{C}_{1}$ and the conditions (i) and (ii) are satisfied. However, the nonzero vector $f=1 \oplus 0$ is in $H^{2}(\mathbb{D}) \oplus \mathbb{C}_{1}$ but $f \otimes_{\mathcal{A}} 1_{z}=0$ for $z \in \mathbb{D}$.

Another question is whether $\operatorname{ker} Q=\{0\}$ is necessary for $\mathcal{R}_{\mathcal{M}}$ to be quasi-free. However, to establish that one would need to exhibit a nonzero vector $h$ in $\mathcal{R}_{\mathcal{M}}$ satisfying $h \otimes_{\mathcal{A}} 1_{\underline{z}}=0$ for every $\underline{z}$ in $\Omega$ assuming $\operatorname{ker} Q \neq\{0\}$. If $h$ is a nonzero vector in $\operatorname{ker} Q$, then $\left(Q \otimes_{\mathcal{A}} 1_{\underline{z}}\right)\left(h \bar{\otimes}_{\mathcal{A}} 1_{\underline{z}}\right)=$ $(Q h) \otimes_{\mathcal{A}} 1_{\underline{z}}=0$. The proof would be completed by showing that $\operatorname{ker}\left(Q \otimes_{\mathcal{A}} 1_{\underline{z}}\right)=\{0\}$ for each $\underline{z} \in \Omega$, where $Q \otimes_{\mathcal{A}} 1_{\underline{z}}: \mathcal{R}_{\mathcal{M}} \otimes_{\mathcal{A}} \mathbb{C}_{\underline{z}} \rightarrow \mathcal{R} \otimes \mathbb{C}_{\underline{z}}$. The module map $Q \otimes_{\mathcal{A}} \underline{1}_{\underline{z}}$ is defined by taking the generating set $\left\{\left(f_{i} \oplus g_{i}\right) \otimes_{\mathcal{A}} 1_{\underline{z}}\right\}$ termwise to the generating set $\left\{g_{i} \otimes_{\mathcal{A}} 1_{\underline{z}}\right\}$. If $k<\infty$, then both of the spaces $\mathcal{R}_{\mathcal{M}} \otimes_{\mathcal{A}} \mathbb{C}_{\underline{z}}$ and $\mathcal{R} \otimes_{\mathcal{A}} \mathbb{C}_{\underline{z}}$ are $k$ - dimensional and the map $Q \otimes_{\mathcal{A}} 1_{\underline{z}}$ is onto. Therefore, the kernel is trivial and the converse is seen to hold. For $k=\infty$, we are unable to conclude that the maps $Q \otimes_{\mathcal{A}} 1_{\underline{1} z}$ have trivial kernels. To proceed further in the $k=\infty$ case, one would need more information on the nature of the bases defined by the $\left\{f_{i}\right\}$ and the $\left\{g_{i}\right\}$ and their relationship to each other. 
One would like to show that $\operatorname{ker} Q=\{0\}$ if $\mathcal{M}$ belongs to class $C_{.0}$ and a proof would seem almost at hand. However, what the argument seems to requires is assuming that the module $\mathcal{M}$ satisfies a stronger condition than that of membership in the class $C_{.0}$. One can complete the proof if in the definition of class $C_{.0}$ the uniform bound on the supremum norm for the sequence in $\mathcal{A}(\Omega)$ is replaced by a uniform bound on the Hilbert module norm in the quasi-free module, but that would seem to be asking too much. Thus it is not clear just what is the relationship between the notions of class $C_{._{0}}$ and weakly regular.

Now we come to our principal result, the existence of resolutions.

THEOREM 1. Every Hilbert module in class (PS) possesses a weak resolution by quasi-free Hilbert modules.

Proof. Let us first consider the finitely generated case. It is enough to show for $\mathcal{M}$ in class (PS) that the kernel of the module map $Q: \mathcal{R}_{\mathcal{M}} \rightarrow \mathcal{M}$ is trivial for $\mathcal{R}$ quasi-free. If $\mathcal{L}$ is a finite dimensional Hilbert module supported at a point $\underline{z}$ in $\Omega$, then module multiplication by $\varphi$ on $\mathcal{L}$ depends only on the values of $\varphi$ and a fixed finite set of partial derivatives of $\varphi$ at $\underline{z}$. Now suppose $Q(k \oplus 0)=0$ for some vector $k \oplus 0$ in $\mathcal{R}_{\mathcal{M}}$, which is the form a vector must have in $\operatorname{ker} Q$. Then there exists a sequence of functions $\left\{\varphi_{i}^{(n)}\right\}$ such that $\sum \varphi_{i}^{(n)} f_{i} \oplus \varphi_{i}^{(n)} g_{i} \rightarrow k \oplus 0$. By the definition of the norm on $\mathcal{M} \oplus \mathcal{R}$, and the fact that $Q(k \oplus 0)=0$, it follows that $\sum \varphi_{i}^{(n)} f_{i} \rightarrow k$ in $\mathcal{M}$ and $\sum \varphi_{i}^{(n)} g_{i} \rightarrow 0$ in $\mathcal{R}$. Since $\mathcal{R}$ is quasifree, it follows that $\sum \varphi_{i}^{(n)} g_{i} \rightarrow 0$ implies $\varphi_{i}^{(n)}(\underline{z}) \rightarrow 0$ and the same is true for evaluation at $\underline{z}$ of the partial derivatives of $\varphi_{i}^{(n)}$, all of which follows by localizing $\mathcal{R}$ with respect to the modules $\mathcal{Q}_{\underline{z}}^{n}$. This, of course, implies that the image $\sum \varphi_{i}^{(n)} g_{i} \otimes_{\mathcal{A}} x \rightarrow 0$ for $x \in \mathcal{L}$ since $\sum \varphi_{i}^{(n)} g_{i} \otimes_{\mathcal{A}} x=\sum g_{i} \otimes_{\mathcal{A}} \varphi_{i}^{(n)}(\underline{z}) x$ and the module action of $\varphi_{i}^{(n)}(\underline{z})$ on the vector $x$ in $\mathcal{L}$ depends only on a fixed number of partial derivatives of the functions at $\underline{z}$. But this implies that the image of $k$ is zero in $\mathcal{R} \otimes_{\mathcal{A}} \mathcal{L}$ and hence by assumption, $k \oplus 0$ is zero or $\operatorname{ker} Q=\{0\}$.

Now suppose $\mathcal{M}$ has infinite rank with generators $\left\{f_{i}\right\}_{i \in \mathbb{N}}$ and let $\mathcal{R}$ be a rank one quasifree Hilbert module over $\mathcal{A}(\Omega)$ with generator $g$. Let $\mathcal{M}_{i}$ be the submodule of $\mathcal{M}$ generated by $f_{i}$ for $i \geq 1$. We can construct a module $\mathcal{R}_{\mathcal{M}_{i}}$ for each $i \geq 1$ with contractive module map $X_{i}: \mathcal{R}_{\mathcal{M}_{i}} \rightarrow \mathcal{M}$ having range dense in $\mathcal{M}_{i} \subseteq \mathcal{M}$. Since each $\mathcal{M}_{i}$ is a submodule of $\mathcal{M}$, it follows that $\mathcal{M}_{i}$ belongs to class (PS) and hence each $\mathcal{R}_{\mathcal{M}_{i}}$ is quasi-free over $\mathcal{A}(\Omega)$ for the basis $\left\{g \oplus f_{i}\right\}$. Moreover, since the bounds for evaluation at $\underline{z}$ on all $\mathcal{R}_{\mathcal{M}_{i}}$ are dominated by the bound on evaluation at $\underline{z}$ on $\mathcal{R}$, this implies that $\mathcal{R}_{\tilde{\mathcal{M}}}=\oplus \mathcal{R}_{\mathcal{M}_{i}}$ is quasi free over $\mathcal{A}(\Omega)$ for the basis $\left\{g \oplus f_{i}: i \geq 1\right\}$. If we define $X: \mathcal{R}_{\tilde{\mathcal{M}}} \rightarrow \mathcal{M}$ such that $X\left(\sum k_{i}\right)=\sum \frac{1}{2^{i}} X_{i} k_{i}$, then $X$ is well-defined and bounded since $\left\|X\left(\sum k_{i}\right)\right\|=\left\|\sum \frac{1}{2^{i}} X_{i} k_{i}\right\| \leq \sum \frac{1}{2^{i}}\left\|X_{i} k_{i}\right\| \leq \sum \frac{1}{2^{i}}\left\|k_{i}\right\| \leq$ $\left\|\sum k_{i}\right\|$. This completes the proof of weak regularity in the case of infinite rank.

Given $X: \mathcal{R}_{1} \rightarrow \mathcal{M}$ with $\mathcal{R}_{1}=\mathcal{R}_{\mathcal{M}}$ or $\mathcal{R}_{\tilde{\mathcal{M}}}$, the kernel of $X_{0}=X$ is a submodule of a quasi-free Hilbert module and hence belongs to class (PS). Thus we can repeat the construction using ker $X_{0}$ in place of $\mathcal{M}$ and continue to obtain $X_{1}: \mathcal{R}_{2} \rightarrow \mathcal{R}_{1}$. We continue the process using ker $X_{i}$ with the process terminating if $\operatorname{ker} X_{i}$ is a quasi-free Hilbert module. In that case one takes the last $\mathcal{R}_{i}$ to be the zero module. Otherwise, one continues the process indefinitely obtaining an infinite resolution.

Note that if $\mathcal{M}$ is finitely generated, then the module $\mathcal{R}_{0}$ can be taken to be finitely generated as well. However, unless one can conclude that the kernels of the $X_{i}$ are finitely 
generated, we can say nothing about the existence of a resolution by finitely generated, quasifree Hilbert modules. The purpose in proving this result for the class (PS) was to obtain this finiteness result. We could have proved the following result directly which includes Theorem 1.

THEOREM 2. Every Hilbert module in class (SM) has a weak resolution by quasi-free Hilbert modules.

Proof. The argument for the infinitely generated case given in the proof of the preceding theorem can be used, once we know that a singly generated Hilbert module in class (SM) is weakly regular. Hence, assume that $\mathcal{M}$ is a singly generated Hilbert module over $\mathcal{A}(\Omega)$ in class (SM) with generating vector $f$, and that $\mathcal{R}$ is a singly generated Hilbert module over $\mathcal{A}(\Omega)$ with generating vector $g$. We need to show that $\operatorname{ker} Q=\{0\}$, where $Q: \mathcal{R}_{\mathcal{M}} \rightarrow \mathcal{R}$. A vector in the kernel of $Q$ must have the form $k \oplus 0$ in $\mathcal{M} \oplus \mathcal{R}$. Moreover, there exists a sequence $\varphi_{n} \in \mathcal{A}(\Omega)$ such that $\varphi_{n} f \oplus \varphi_{n} g$ converges to $k \oplus 0$, and hence $\varphi_{n} f$ converges to $k$ and $\varphi_{n} g$ converges to 0 .

Now consider the vector $S k=\lim S\left(\varphi_{n} f\right)=\lim \left(\varphi_{n} f \otimes_{\mathcal{A}(\Omega)} g\right)=\lim \left(f \otimes_{\mathcal{A}(\Omega)} \varphi_{n} g\right)=$ $f \otimes_{\mathcal{A}(\Omega)}\left(\lim \varphi_{n} g\right)=0$.

Therefore, the assumption that $\mathcal{M}$ is smooth for $\mathcal{R}$ and $g$ implies $k=0$ or $\operatorname{ker} Q=\{0\}$ which completes the proof.

We can also show that almost compactly supported Hilbert modules have weak resolutions.

THEOREM 3. Every almost compactly supported Hilbert module over $\mathcal{A}(\Omega)$ has a weak resolution by quasi-free Hilbert modules.

Proof. As before, it is sufficient to show that an almost compactly supported Hilbert module is weakly regular. Let $\left\{f_{i}\right\}_{i \in \mathbb{N}}$ be a set of compactly supported vectors in $\mathcal{M}$ that spans $\mathcal{M}$, and let $\mathcal{M}_{i}$ be the submodule of $\mathcal{M}$ generated by $f_{i}$. Let $\mathcal{R}$ be the Bergman module for $\Omega$ with the function 1 as a basis and let $\mathcal{R}_{\mathcal{M}_{i}}$ be the module constructed from $\mathcal{R}$ and $\mathcal{M}_{i}$. Further, let $X_{i}$ be the map from $\mathcal{R}_{\mathcal{M}_{i}}$ to $\mathcal{M}$ with range dense in $\mathcal{M}_{i}$. If we can show that each $\mathcal{R}_{\mathcal{M}_{i}}$ is quasi-free, then we can complete the proof as we did for theorem 1 . Fix an $i \geq 1$. If $k \oplus 0$ is in the kernels of the corresponding $Q_{i}$ from $\mathcal{R}_{\mathcal{M}_{i}}$ to $\mathcal{R}$, then there is a sequence of functions $\varphi_{n} \in \mathcal{A}(\Omega)$ such that $\varphi_{n} f_{i} \oplus \varphi_{n} 1$ converges to $k \oplus 0$. In the Bergman space, it follows that this implies the sequence $\left\{\varphi_{n}\right\}_{n \in \mathbb{N}}$ converges uniformly to zero on compact subsets of $\Omega$. But since the vector $f_{i}$ is compactly supported, there exists a compact subset $X$ of $\Omega$ and a constant $\beta$ such that $\left\|\varphi_{n} f_{i}\right\|_{\mathcal{M}} \leq \beta\left\|\varphi_{n}\right\|_{X}\left\|f_{i}\right\|_{\mathcal{M}}$ and hence $k=\lim \varphi_{n} f_{i}=0$. Thus $\operatorname{ker} Q_{i}=\{0\}$ which completes the proof.

The purpose of this paper is to establish the existence of weak resolutions under hypotheses as general as possible. Unfortunately, the present theorems are not completely satisfactory in that they do not provide an intrinsic characterization of those Hilbert modules for which weak resolutions exist. In discussing this matter further, let us focus on the question of when a Hilbert module is weakly regular. As the proofs indicate, weak regularity rests on the module being supported in some sense on the open set $\Omega$. Further consideration of the module obtained as the quotient of the Hardy module over $\mathcal{A}(\mathbb{D})$ by a submodule determined by a singular inner function shows that weak regularity does not imply almost compactly supported. Also, this example shows that while almost compactly supported implies class 
$C_{.0}$, the converse is false. Thus weak regularity lies somewhere between almost compactly supported and class $C_{.0}$, and perhaps coincides with the latter.

Another question is to determine the class of modules for which exact resolutions exist. The construction presented above seems unlikely to yield resolutions since that would mean showing that the module map $P: \mathcal{R}_{\mathcal{M}} \rightarrow \mathcal{M}$ is onto. Clearly that depends on having greater control on comparisons between the norms of the vectors of the form $\sum \varphi_{i} f_{i}$ and $\sum \varphi_{i} g_{i}$. Although one could take $\mathcal{R}$ to be the Bergman space, as we have at various junctures above, the inequalities one would need are not available, in general. A different construction, based on the one given in (Chapter 5, 24) might be used to show that a compactly supported $\mathcal{M}$ is regular but the details are not all clear. Recall that we do know that a regular Hilbert module lies in class $C_{.0}$ but, unfortunately, not the converse. Understanding whether class $C_{.0}$ implies that a Hilbert module is weakly regular or regular are extremely important in continuing this approach.

Given that we know so little about regularity, it would seem almost presumptuous to even introduce the notion of strong regularity and a modicum of experience would suggest that it hardly ever happens. However, the resolution provided by the Sz.-Nagy-Foias model is strongly regular. Moreover, if one were to consider this question without appealing to the model theory, we believe it might be hard to make the right guess. For example, it would seem unlikely for there to be a strong resolution of the Bergman module over the disk by a direct sum of Hardy modules, but there is, albeit one of infinite multiplicity. In a different direction, consider the second resolution of $\mathbb{C}_{\underline{0}}$ given in section 2 . While the maps are onto, they are not partial isometries. We presented this resolution in the form given there because that is the most natural way. However, with minor changes in the norms on the resolving modules, one obtains a strong resolution. Here are the details.

Recall one maps the Hardy module $H^{2}\left(\mathbb{D}^{2}\right)$ over the bi-disk algebra $\mathcal{A}\left(\mathbb{D}^{2}\right)$ onto $\mathbb{C}_{\underline{0}}$ which is a partially isometric map. Then one maps the direct sum of two copies of $H^{2}\left(\mathbb{D}^{2}\right)$ to $H^{2}\left(\mathbb{D}^{2}\right)$ by the map $X(f \oplus g)=z_{2} f-z_{1} g$ which is not a partial isometry. However, suppose one changes the norms on the Hardy modules so that on the first one, if $a_{i, j}$ are the Taylor coefficients of $f$, then we multiply the $a_{i, 0}$ by a factor $\sqrt{1 / 2}$ and on the second one, if $b_{i, j}$ are the Taylor coefficients of $g$, then we multiply the $b_{0, j}$ by a factor $\sqrt{1 / 2}$. The resulting Hilbert modules are still quasi-free over $\mathcal{A}\left(\mathbb{D}^{2}\right)$ since both changes yield equivalent norms. However, now $X$ is a partial isometry. Now, the last non-zero term in the resolution is a copy of $H^{2}\left(\mathbb{D}^{2}\right)$ with the map $Y$ defined by $Y f=z_{1} f \oplus z_{2} f$. Here, one wants to multiply all the Taylor coefficients of a vector $f$ in $H^{2}\left(\mathbb{D}^{2}\right)$ by a factor $\sqrt{1 / 2}$ to obtain an equivalent Hilbert module which is quasi-free and which makes $Y$ into a partial isometry. Thus, $\mathbb{C}_{\underline{0}}$ has a strong resolution.

The question of whether or not resolutions or strong resolutions exist is not merely academic for the following reason. If a Hilbert module $\mathcal{M}$ is regular, then there exists a quasi-free Hilbert module $\mathcal{R}$ and a module map $X$ from $\mathcal{R}$ onto $\mathcal{M}$. If $\operatorname{ker} X=\{0\}$, then $\mathcal{M}$ is similar to the quasi-free module $\mathcal{R}$ and hence, $\mathcal{R}$ is itself quasi-free. Otherwise, we may assume there is a nontrivial kernel. If a full resolution exists, then there are nontrivial kernels and we can continue or the resolution stops and has finite length. This is the situation in commutative algebra and one should expect in such a case to be able to extract information about $\mathcal{M}$ from the resolution using the extension of techniques from commutative algebra.

There is another issue which it is convenient to raise at this time, namely, are resolutions finite? In general, the answer must be negative. However, one would expect that there is a 
large class of Hilbert modules for which that is the case. A related question is whether the kernel of a module map $X: \mathcal{R} \rightarrow \mathcal{M}$ is finitely generated. Again, one would assume that this is the case for a large class of Hilbert modules when both $\mathcal{M}$ and $\mathcal{R}$ are finitely generated, but results seem to be difficult [39]. The questions we are raising here, of course, concern coherence-like properties of the spectral sheaf $\operatorname{Sp}(\mathcal{M})$. If one replaces Hilbert modules by Frechet modules, then there is a lot of work on these questions (cf. [27]). Connecting the two approaches, where Hilbert spaces are used on the one hand or Frechet spaces on the other, seems difficult. Our point of view has been that the appropriate domain for multivariate operator theory is Hilbert space but any final assessment must rest on the results obtained and their utility.

\section{Usefulness of Resolutions}

This paper has been devoted to showing the existence of quasi-free resolutions of Hilbert modules. There would be little point in constructing such resolutions if they were not useful in studying the original modules. In this section we want to sketch some ways in which resolutions have been useful and could be useful in the general study of Hilbert modules.

As we have indicated, one can re-interpret the model of Sz.-Nagy and Foias as a resolution. Thus, in principle, one could argue that all of model theory could be taken over to the context of resolutions but that would be an exaggeration. Much of the theory depends on the rich interplay of function theory, functional analysis, and Fourier analysis which come together on the unit disk with boundary the unit circle. Also, some of the theory depends on the fact one has a strong resolution in our language rather than just a resolution or even a weak

resolution. Still the characterization of at least one basic notion carries over, that of the spectrum.

Recall that the resolution for a contractive Hilbert module $\mathrm{H}$ of class $C_{.0}$ has the form:

$$
0 \longleftarrow \mathcal{H} \longleftarrow H_{\mathcal{E}}^{2}(\mathbb{D}) \stackrel{X}{\longleftarrow} H_{\mathcal{E}^{*}}^{2}(\mathbb{D}) \longleftarrow 0
$$

where $X$ is an isometric module map. If one localizes $X$ by $C_{z}$, one can show that $X \otimes_{\mathcal{A}(\mathbb{D})}$ $1_{z}=\Theta(z)$, where $\Theta$ is the characteristic operator function of Sz.-Nagy and Foias. In general model theory, one knows that $\Theta$ is an operator-valued inner function, that is, it has radial limits a. e. on the circle which are unitary operators from $\mathcal{E}^{*}$ to $\mathcal{E}$. Our interest is in the connection of $\Theta$ with the spectrum which one knows is the union of the set of points in $\mathbb{D}$ at which $\Theta(z)$ fails to be invertible plus the closed subset of the boundary on which $\Theta$ fails to have an analytic continuation. One can show directly from the exactness of the resolution that the spectrum inside $\mathbb{D}$ consists of the points at which the localization $X \otimes_{\mathcal{A}(\mathbb{D})} 1_{z}$ fails to be invertible and, indeed, that the nature of the spectrum of the operator defined by module multiplication by $z$ is that same as that of $\Theta(z)$. The details of this calculation are given in [24].

Now suppose we have a weak resolution of a Hilbert module $\mathcal{M}$ over $\mathcal{A}(\Omega)$. One can calculate the spectrum of the module which is defined using the Taylor spectrum (cf. [24]), in terms of the resolution. Moreover, one can determine the nature of the spectrum, that is, the nature of the lack of exactness of the Koszul complex a la Taylor. One should compare a recent paper by D. Greene [26] in which he does something similar for modules over an algebra of holomorphic functions but one which is not a function algebra. In both cases, the behavior of the Hilbert module on the boundary would have to be investigated using different techniques. As we indicated above, on the disk the determination of the full spectrum 
involves the notion of analytic continuation. Although, there are other characterizations, none involve strictly algebraic notions.

There is another class of invariants for Hilbert modules of a very different nature, associated with complex geometry. In the late seventies, M. Cowen and the first author introduced a class of operators which have a hermitian holomorphic bundle associated with them. Moreover, they showed that the geometric invariants of the bundle form a complete set of unitary invariants for the operator. This approach was extended by Curto and Salinas [13] to the case of commuting n-tuples of operators, and by X. Chen and the first author 9] to certain classes of Hilbert modules. The latter class includes the quasi-free ones and the associated spectral sheaves are the corresponding hermitian holomorphic vector bundles. Thus quasifree Hilbert modules can be characterized up to unitary equivalence by the curvature and a finite set of partial derivatives of curvature over $\Omega$. In a series of papers [20, [22], 21], [23], the authors along with Verughese, have related the geometrical invariants for Hilbert modules in a resolution. In particular, one shows for the quotient module defined by the functions in a quasi-free module $\mathcal{R}$ that vanish to some order along a hypersurface, that the geometric invariants for the spectral sheaf for the quotient are determined by those for the quasi-free sheaf in the form of longitudinal curvature, transverse curvature and a second fundamental form involving an appropriate jet bundle.

One can formulate relations such as the above for weak resolutions although the formulas and proofs will involve, ultimately, an extension of techniques related to the work of HarveyLawson [28] as well as to that of Demailley [16]. Some very simple cases have been established but there is much to do and the possibility for relating unitary invariants for a module to those of a weak resolution seem promising.

\section{REFERENCES}

[1] B. Abrahamse and R. G. Douglas, Operators on multiply connected domains, Proc. Royal Irish Acad., 74A (1974), 135 - 141.

[2] O. P. Agrawal and N. Salinas, Sharp kernels and canonical subspaces Amer. J. Math., 110 (1988), 23 47.

[3] T. Ando, On pair of commuting contractions, Acta. Sci. Math., 24 (1963), 88 -90.

[4] W. Arveson, Subalgebras of $C^{*}$ algebras, Acta Math., 123 (1969), 141 - 224.

[5] W. Arveson, Subalgebras of $C^{*}$ algebras II, Acta Math., 128 (1969), 271 - 308.

[6] W. Arveson, Subalgebras of $C^{*}$ algebras III, Multivariable operator theory, Acta Math., 181 (1998), 159 $-228$.

[7] S. Axler, J. McCarthy, and D. E. Sarason, Holomorphic Spaces, Math. Sci. Res. Inst. Publ., 33, Cambridge University Press, Cambridge, 1998.

[8] A. Beurling, On two problems concerning linear transformations in Hilbert space, Acta Math., 81 (1949), $239-255$.

[9] X. Chen and R. G. Douglas, Localization of Hilbert Modules, Michigan Math. J., 39 (1992), 443 - 454.

[10] A. Connes, Noncommutative geometry Academic Press Inc., 1994. xiv+661 pp.

[11] M. Cowen and R. G. Douglas, Complex geometry and operator theory, Acta Math., 141 (1978), $187-261$.

[12] M. Cowen and R. G. Douglas, On operators possessing an open set of eigenvalues, Memorial conf. for Féjer-Riesz, Budapest, Colloq. Math. Soc. J. Bolyai, (1980), 323 - 341.

[13] R. E. Curto and N. Salinas, Generalized Bergman kernels and the Cowen-Douglas theory, Amer J. Math., 106(1984), 447 - 488.

[14] K. R. Davidson, Nest Algebras, Pitman Research Notes in Mathematics Series, 191, Longman, Harlow, England, 1988.

[15] K. R. Davidson, Free semigroup algebras. A survey, IN "Systems, approximation, singular integral operators, and related topics (Bordeaux, 2000)", 209-240, Oper. Theory Adv. Appl., 129, Birkhuser, Basel, 2001. 
[16] Jean-Pierre Demailly, Multiplier ideal sheaves and analytic methods in algebraic geometry, ICTP School, April - May, 2000.

[17] R. G. Douglas, Models and resolutions for Hilbert modules, Contemporary Mathematics, 185, 109 - 131.

[18] R. G. Douglas, On Silov resolutions of Hilbert modules, IN "Operator Theory: Advances and Applications", vol. 28, Birkhauser Verlag Basel,(1988), pp 51 - 60.

[19] R. G. Douglas, Invariants for Hilbert Modules, Operator theory/ Operator Algebras and Applications, Proc. Symp. Pure Math., 51(1990), 179 - 196.

[20] R. G. Douglas and G. Misra, Geometric invariants for resolutions of Hilbert modules, IN "Operator Theory: Advances and Applications", Vol. 104, 83-112, Birkhäuser, Basel, 1998.

[21] R. G. Douglas, G. Misra and C. Varughese, On quotient modules - The case of arbitrary multiplicity, J. Functional Anal., 174 (2000), 364 - 398.

[22] R. G. Douglas and G. Misra, On quotient modules, IN "Operator Theory: Advances and Applications," Vol. 127, 203 - 209, Birkhäuser, Basel, 2001.

[23] R. G. Douglas, G. Misra and C. Varughese, Some geometric invariants from resolutions of Hilbert modules, IN "Operator Theory: Advances and Applications," Vol. 129, 241 - 270, Birkhäuser, Basel, 2001.

[24] R. G. Douglas and V. I. Paulsen, Hilbert Modules over Function Algebras, Longman Research Notes, $217,1989$.

[25] R. G. Douglas, V. I. Paulsen, C.-H. Sah and K. Yan, Algebraic reduction and rigidity for Hilbert modules, Amer. J. Math. 117 (1995), no. 1, 75-92.

[26] D. C. V. Greene, On free resolutions in multivariable operator theory, preprint.

[27] J. Eschemeier and M. Putinar, Spectral Decompositions and Analytic Sheaves, London Math. Soic., Clarendon Press, Oxford, 1996

[28] F. R. Harvey and H. B. Lawson, A theory of characteristic currents associated with a singular connection, Asterisque, 213 (1993), 1 - 268.

[29] R. V. Kadison and I. M. Singer, Triangular operator algebras, Fundamentals and hyperreducible theory, Amer. J. Math., 82 (1960), 227-259. operators, Ann. Math. (2) 37 (1936) 116 - 229.

[30] F. Murray and J. von Neumann, On rings of operators, Ann. Math., (2), 37 (1936) 116 - 229.

[31] F. Murray and J. von Neumann, On rings of operators II, Trans. Amer. Math. Soc., 41 (1937), no. 2, 208-248.

[32] F. Murray and J. von Neumann, On rings of operators IV, Ann. of Math., (2), 44 (1943), 716-808.

[33] B. Sz-Nagy and C. Foias, Harmonic Analysis of operators on Hilbert space, North Holland, 1970.

[34] J. von Neumann, On rings of operators III Ann. of Math., 41 (1940), 94-161.

[35] J. von Neumann, Eine Spektraltheorie fr allgemeine Operatoren eines unitren Raumes, Math. Nachr., 4 (1951), 258-281.

[36] N. K. Nikolski, Treatise on the shift operator: Spectral function theory, Grundlehren der mathematischen Wissensschaften, 273, Springer Verlag, Berlin, 1985.

[37] V. I. Paulsen, Completely bounded maps and dilations, Pitman Research Notes in Mathematics, 146 (1986), London.

[38] G. Popescu, Universal operator algebras associated to contractive sequence of non-commuting operators., J. London Math. soc., 58 (1998), 469 - 479.

[39] R. Yang, Hardy Modules, PhD Thesis, SUNY Stony Brook, May 1998.

Texas A\&M University, College Station, Texas 77843

E-mail address: rgd@tamu.edu

Indian Statistical Institute, RV College Post, Bangalore 560059

E-mail address: gm@isibang.ac.in 